\title{
CRYSTALLOCHEMICAL CHARACTERIZATION OF THE PALYGORSKITE AND SEPIOLITE FROM THE ALLOU KAGNE DEPOSIT, SENEGAL
}

\author{
E. García-R $\bullet m e r \bullet^{1 *}$, M. Suárez ${ }^{2}$, J. Santarén ${ }^{3}$ and A. Alvarez ${ }^{3}$ \\ ${ }^{1}$ Departamento de Cristalografía y Mineralogía, Universidad Complutense de Madrid, E-28040 Madrid, Spain \\ 2 Departamento de Geología, Universidad de Salamanca, E-37008 Salamanca, Spain \\ ${ }^{3}$ TOLSA Cra Vallecas-Mejorada del Campo, km 1600, 28031 Madrid, Spain
}

\begin{abstract}
The Allou Kagne (Senegal) deposit consists of different proportions of palygorskite and sepiolite, and these are associated with small quantities of quart $z$ and $X$-ray amorphous silica as impurities. No pure palygorskite or sepiolite has been recognized by X-ray diffraction. Textural and microtextural features indicate that fibrous clay minerals of the Allou Kagne deposit were formed by direct precipitation from solution. Crystal-chemistry data obtained by analytical/ransmission electron microscopy (AEM/ TEM) analyses of isolated fibers show that the chemical composition of the particles varies over a wide range, from a composition corresponding to palygorskite to a composition intermediate between that of sepiolite and palygorskite, but particles with a composition corresponding to sepiolite have not been found. Taking into account the results from selected area electron diffraction and AEM-TEM, fibers of pure palygorskite and sepiolite have been found but it cannot be confirmed that all of the particles analyzed correspond to pure palygorskite or pure sepiolite because both minerals can occur together at the crystallite scale. In addition, the presence of $\mathrm{Mg}$-rich palygorskite and very $\mathrm{Al}$-rich sepiolite can be deduced.

It is infrequent in nature that palygorskite and sepiolite appear together because the conditions for simultaneous formation of the two minerals are very restricted. The chemical composition of the solution controls the formation of the Allou Kagne sepiolite and palygorskite. The wide compositional variation appears as a consequence of temporary variations of the chemical composition of the solution.
\end{abstract}

Key Words-AEM-TEM, Allou-Kagne Deposits, Crystal Chemistry, Palygorskite, SAED, Senegal, Sepiolite

\section{INTRODUCTION}

Sepiølite and palygerskite are fibrøus clay minerals with many industrial applications due to their structural and physicechemical preperties. They are used as abserbents and as adserbents (cat litter, separation of gases, filters, etc.); they have rhe@legical preperties (drilling mud on salt water, pharmacy, paint, cosmetic, etc.) and have numerous other uses (Alvarez, 1984; Jønes and Galán, 1988).

The structure of both sepiølite and palygerskite contains ribbens of 2:1 phyllesilicates linked by periedic inversion of the apical exygen of the continu tetrahedral sheet (every six atøms of Si for sepiolite and every four for palygorskite). Sepielite is a tri-ctahedral mineral with eight pessible ectahedral pesitiens per half unit-cell, and all are eccupied. The structural formula of sepiolite is $\mathrm{Si}_{12}{ }_{30} \mathbf{M g}_{8}$ $\left(\bullet \mathrm{H}_{4}\left(\mathrm{H}_{2}\right)_{4} . n \mathrm{H}_{2} \bullet\right.$ (Brauner and Preisinger, 1956). The octahedral sheet is discontinuous and terminal cations must complete their coordination sphere with

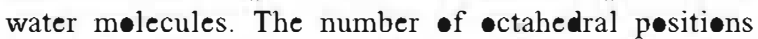
(per half unit-cell) in palygerskite is five, although it does not seem possible that all can be filled (Serna et al.,

* E-mail address of corresponding author: mromero@geo.ucm.es
1977). In mest palygerskites the number of eccupied positions ranges from four to five, and only a few cases seem to be completely diectahedral with the structural formula $\mathrm{Si}_{8} \bigcirc_{20} \mathrm{Al}_{2} \mathrm{Mg}_{2}(\bullet)_{2}\left(\mathrm{H}_{2}\right)_{4} \cdot 4 \mathrm{H}_{2} \bullet$ (Bradley, 1940).

Palygerskite and sepiølite form in marine or cøntinental sedimentary envirenments. Both minerals may be formed by direct precipitation from waters with a large degree of salinity cøming frøm strøngly weathered centinental areas (Castillø, 1991), and beth minerals are frequently associated with lacustrine facies in continental sediments where they form frem sølutions $\bullet$ by diagenetic transformation (Weaver, 1984; Jones and Galán, 1988; Chahi et al., 1997). Palygerskite is especially commen in calcretes related to edaphic processes affecting sediments (Singer and Norrish, 1974; Watts, 1980; Singer, 1984; Verrecchia and Le Coustumer, 1996). Palygorskite and sepiølite can als be formed as direct precipitates $\bullet$ as a replacement preduct frøm hydrethermal sølutiøns (Tien, 1973; Haji-Vassiløu and Puffer, 1975; López Galinde et al., 1996; Kamineni

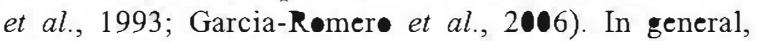
their genesis can be related to transformation precesses from previous silicates (Yaalon and Wieder, 1976; Suárez et al., 1994; Tørres-Ruiz et al., 1994; LøpezGalinde et al., 1996) or to direct precipitation frøm sølutions (Singer and Norrish, 1974; Watts, 1976; Estéøule-Chøux, 1984). 
Palygorskite is more abundant than sepiolite and although the two minerals sometimes appear together this is not often the case. There are few references in the literature in which sepielite and palygorskite eccur in the same or adjacent localities. For example, they are described together in the following Spanish deposits: Taj• Basin, (Lequey et al., 1995; Galán and Castill•, 1984), Tabladill• (Mattín Pozas et al, 1981); Lebrija (Galán and Ferrer•, 1982); and als• in central and central-southern Tunisia (Zaaboub et al, 2005), in the Serinhisar-Acipayam Basin (Turkey) (Akbulut and Kadir, 203), and in the deep-sea mid-Atlantic ridge, -f hydrothermal origin (Bowles et al,, 1971). In the Allou Kagne deposit, sepiolite and palygorskite appear together. This is an important deposit of special clays.

The aim of this work is the mineralogical and crystallochemical characterization of the palygorskite and sepiolite from the Alløu Kagne deposit. The relationships between the two minerals have been studied, both from genetic and compositional viewpoints.

\section{MATERIALS AND METHODS}

\section{Materials}

The palygorskite and sepiolite studied were obtained from the Allou Kagne (Senegal) deposit which is located $\sim 1 \mathrm{~km}$ from the town of Thiès on the road from akar to Thiès (Figure 1). This deposit has been known since the second half of the $20^{\text {th }}$ century. Millot (197) reported it and other deposits of fibrous clays in this region to be of miner economic interest. It is located in the Senegal-Mauritania basin and its genesis is related to sedimentation in an epicontinental marine environment during the Paleogene.

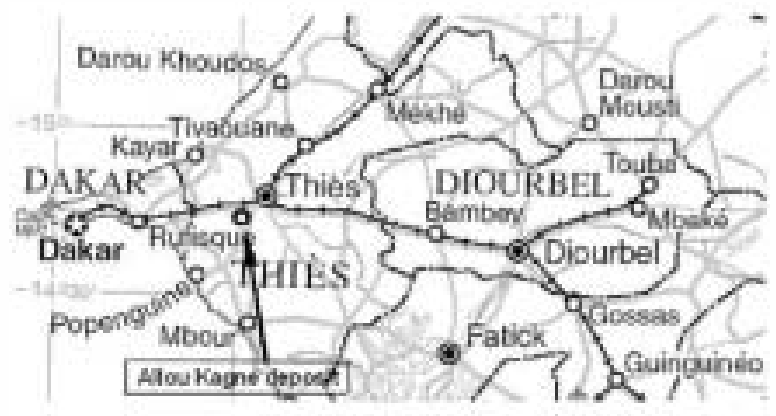

Figure 1. Map of Senegal, shewing the Alløu-Kagne depesit.

Palygorskite and sepiolite usually appear in horizental layers, interbedded with carbonates (calcite and døl-mite) and, in some cases, accessory minerals like quartz and opal A. The fibrous clay layers typically consist mainly of palygorskite intermingled with variable miner quantities of sepielite, are of Lower Eøcene age, and appear over Paleocene karstified limestones (Figure 2). The bottom of the deposit consists of glauconitic sands containing phosphate and carbonate. The section rich in palygorskite and minor sepiolite is divided int two different zones, the lower being the richest in carbonates $(<3 \%)$ and containing massive layers, $\bullet$ chre in color, up to $8 \mathrm{~m}$ thick. The upper zone of the mineralized section is the purest in terms of content - f clay minerals. Parallel laminations in the clay levels are white or beige, and show black and orange spots. In some places, silicified levels are intercalated. The total thickness is rarely $>20 \mathrm{~m}$. Between both zones, a thin level of sandstone may appear. This level is $\sim 1 \mathrm{~cm}$ thick, is silicified and carbonated, and is a guide level. Samples coming from the upper-zone level were chosen

\begin{tabular}{|c|c|c|c|c|c|}
\hline & & pisechanow & MT & $\leqslant \infty 0_{1}$ & sorea \\
\hline gevirwiukr & $x_{x-1}+2$ & $\begin{array}{l}\text { Santatino } \\
\text { Lakrik an. }\end{array}$ & nz & & \\
\hline \multirow{4}{*}{$\begin{array}{l}\text { towe } \\
\text { tocont }\end{array}$} & \multirow{4}{*}{$\frac{\alpha}{-\alpha x}$} & 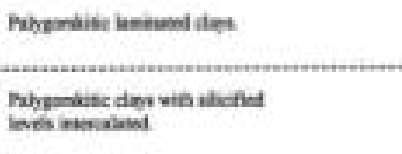 & 21 & $\infty$ & \multirow[t]{2}{*}{19600} \\
\hline & & 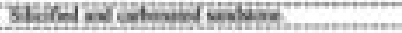 & Fi & & \\
\hline & & 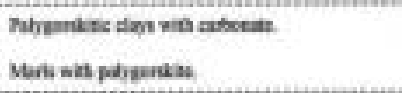 & - & $20-30$ & $<100$ \\
\hline & & 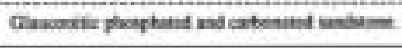 & & & \\
\hline DatzoceNe & P.1. & Kandod lenowar & & $=\infty$ & \\
\hline
\end{tabular}

Figure 2. Schematic stratigraphic section of the Alløu-Kagne depesit. M.T. - maximum thickness (m). W.A. - water abserption. 
for this mineraløgical and crystalløchemical study. The clay beds of ecønmic interest range frem 4 to $20 \mathrm{~m}$ thick, with $\bullet$ verburden that varies from to $2 \mathrm{~m}$. The preven reserves $\bullet$ the depesit are $25 \mathrm{Mt}$ with inferred resøurces of $>60 \mathrm{Mt}$.

T• the north of the area studied, in which the lithølogical series is more complete, sediments from Upper Eøcene and Oligecene or Miø-Pliøcene appear -ver the palygerskite section. These sediments are compesed mainly of phesphated sediments that have formed majør depesits of phosphates which are currently mined.

Quaternary silica sands and laterites, and in some cases marly $\bullet$ clayish søil, appear at the top. In the

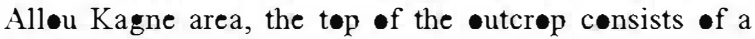
lateritic søil that can reach $20 \mathrm{~m}$ thick in søme places $\bullet$, -nly a few meters in •thers. Three series of vertical faults have caused sub-vertical displacements of the mineralized bed and have determined variations in the compesition and the thickness of the Quaternary sediments.

The clay levels studied show parallel lamination as a consequence of sedimentary $\bullet$ rigin, and hand specimens exhibit a clear planar structure. The sedimentary structure of the samples was taken int account during their study. Thin layers, $\sim 1 \mathrm{~mm}$ thick, were separated to study small-scale compesitional variations. A mineraløgical and crystallechemical characterization of the samples at this scale was als• carried •ut.

\section{Methods}

Mineralıgical characterization was performed by X-ray diffraction (XRD) using a Siemens D50 XRD diffractometer with $\mathrm{CuK} \alpha$ radiation and a graphite

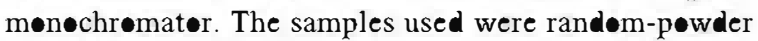
specimens which were scanned frøm 2 to $65^{\circ} 2 \theta$ at $0.05^{\circ} 20 / 3$ s to determine the mineral $\bullet$ gical compesition. $\mathrm{X}$-ray diffraction patterns frem pøwdered individual and contigueus layers of hand specimens were recorded in -rder to determine whether sepiølite and palygerskite are cøncentrated in different layers $\bullet$ whether both minerals always appear together in similar propertions.

Particle morphølegy and textural relationships were established by scanning electrøn microscepy (SEM) and transmissiøn electrøn micrøscopy (TEM). The •bservatiøns were performed using a JEOL JSM 6400 micr scope, operating at $20 \mathrm{kV}$ and equipped with a Link System energy dispersive X-ray (EX) micreanalyzer. Prior to examination by SEM, freshly fractured surfaces - representative samples were air dried and coated with $\mathrm{Au}$ under vacuum. The TEM •bservatiøns were perførmed by depositing a drøp of diluted suspension -n a microscepic grid with colledien. Selected area electron diffraction (SAED) images and chemical

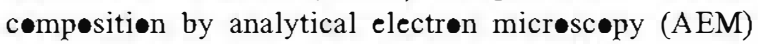
were ebtained by TEM, in pure samples, using a JE L $2000 \mathrm{FX}$ micrescepe equipped with a double-tilt sample holder (up to a maximum of $\pm 45^{\circ}$ ) at an acceleration veltage of $200 \mathrm{kV}$, with $\bullet .5 \mathrm{~mm}$ zeta-axis displacement

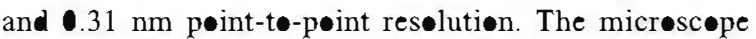
incerpørates an OXFORD ISIS EDX spectrometer $(136 \mathrm{eV}$ reselution at $5.39 \mathrm{keV})$ and has its •wn søftware for quantitative analysis. F॰r AEM analyses, four representative samples with intermediate contents -f both minerals, all corresponding to the upper zone of the depesit were chesen. One representative sample was separated int six layers, millimeters thick, and the new samples were named from AKA-AKF. As it was found that there is a pregressive variation in the relative prepertiøns of clay minerals, frem the richest in palygerskite (AKA) to the richest in sepielite (AKF), the two extreme samples were chesen for the AEM study. Structural formulae were calculated noting that the ideal formula contains 21 and 32 exygens for palygerskite and sepielite, respectively, in the dehydrated and dehydrexylated structure per half unit-cell (Bailey, 1980). All the Fe present was considered as $\mathrm{Fe}^{3+}$ (•wing to the limitation of the technique), but the possible existence of $\mathrm{Fe}^{2+}$ cannot be ruled out.

\section{RESULTS}

\section{Mineralogical composition}

As already stated, raw samples are compesed of different prepertions of sepiølite and palygerskite, and they have small quantities of quartz and X-ray amorphøus silica as impurities. When several layers $\bullet$ a hand specimen have been studied separately, different prøprtiøns of sepiølite and palygorskite have been found in each layer, and the propertion of both minerals varies between contigueus planes. Figure 3 shøws the XRD

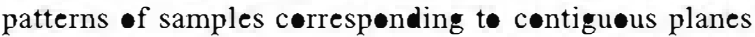
which are $1 \mathrm{~mm}$ thick $\bullet$ btained frøm a laminar sample $0.5 \mathrm{~cm}$ thick. The progressive variation in the percentages of sepielite and palygerskite can be •bserved. N• pure sepielite or palygerskite has been found.

\section{Textural and microtextural features}

The combined SEM •f raw samples and TEM •f dispersed samples have confirmed the characteristic

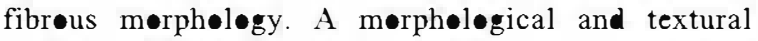
study by SEM indicates that samples are compesed of fibers riented according to the lamination, with the axis of the fibers pointing in all directions (Figure 4a), forming well defined planes, corresponding to their

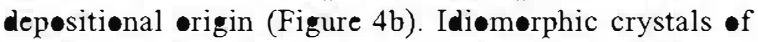
apatite can be observed ameng the fibers. Although sepielite and palygerskite appear together even at this scale, as indicated by XRD, differences in size •r

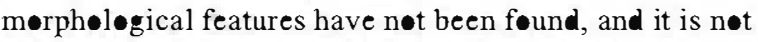
possible to distinguish the two minerals in these samples by SEM •r TEM. The aggregate grøwth •f individual fibers forming planes does n॰t permit measurement of the precise length of these individual crystals, but it is 


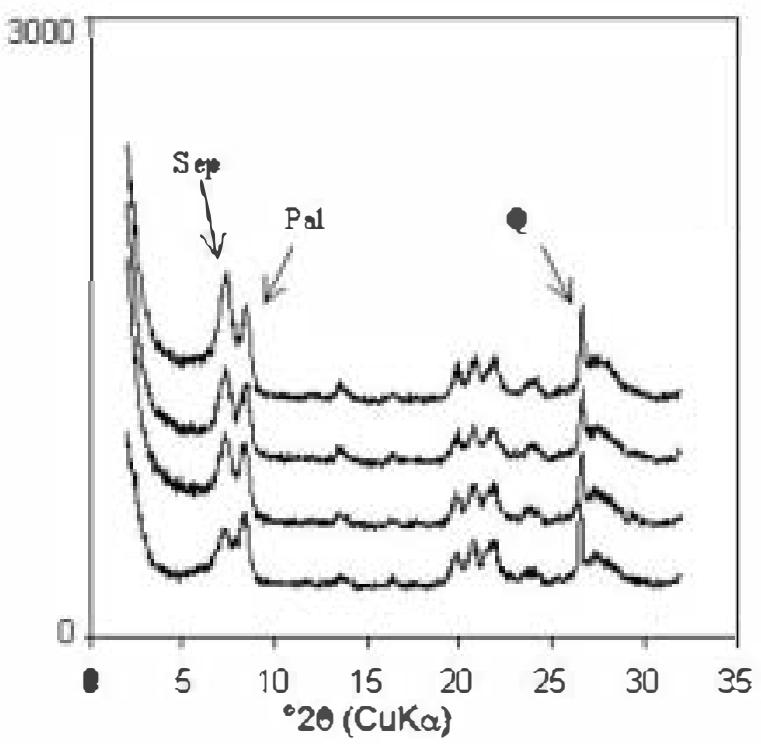

Figure 3. XRD patterns corresponding to contigueus mm-thick layers. Prøgressive change in the prepertions f f the tw fibrous minerals, sepielite (Sep) and palygorskite (Pal), can be seen. Q: quartz.

possible to affirm that the fibers are generally $>1 \mu \mathrm{m}$ in length. From a textural point of view, it is possible to -bserve that the pores are scarce and millimetric in size, because the fibers form planar surfaces and create interfiber pores of $<100 \mathrm{~nm}$ in width.

The characteristic fibrous morph $\bullet$ gy $\bullet$ b both palygorskite and sepiolite can als be observed by TEM. The fibers are $>1 \mu \mathrm{m}$ long (Figure $5 \mathrm{c}$ ), although size can be influenced by the dispersion procedure which can break the fibers. Groups of fibers are disposed in parallel arrangement forming bundles which correspond to the fibers seen by TEM. When the samples were chesen from different and contiguous layers, n॰ differ- ences in size or morph $\bullet$ gical features were found.

Likewise, the SAED patterns of elongated bundles of fibers confirmed the close relationship between sepiolite and palygorskite crystals. Although mest diffraction pattems of is lated fibers correspond to sepiolite or palygorskite crystals (Figure 5a,b), the same diffraction pattems of sepiolite and palygorskite almost parallel in the same fiber have als been observed. Spots correspending to reflections of both sepiolite and palygorskite (12 $\dot{\mathrm{A}}$ and $10.5 \dot{\mathrm{A}}$ ) appear together, as shown in Figure 5d. This means that both minerals coexist at crystallite scale.

\section{Crystallochemical characterization}

Ninety nine TEM pøint analyses of is lated fibers corresponding to different samples were carried out. The chemical compositions of the particles vary between very wide exremes (Table 1). Samples were selected taking inte account the results btained from XR study including the richest in palygorskite and sepiolite. In Figure 6 , the results $\bullet$ btained for the particles analyzed are plotted together with those corresponding to 'theoretical formulae' of palygorskite and sepiolite. The points are distributed on the graph over a very wide range, from palygorskite to sepiolite composition. There are a few analyses with the rati $\mathrm{Si}_{2} \mathrm{Mg}$ and $\mathrm{Al}_{2} \bullet_{3}+\mathrm{Fe}_{2} \bullet_{3}$ similar to that corresponding te palygorskite. However, by contrast, no analysis corresponding to a pure sepiolite composition was found.

According to bibliographic references related to the chemical compesition of fibrous clay minerals, a compositional gap occurs between the two exwemes. The rioctahedral exreme is sepiolite, and the dioctahedral extreme is palygorskite (Martín Vivaldi and Cane, 1956; Paquet et al., 1987; Galán and Carreter•, 1999). In this paper, the structural formulae for all analyzed particles were calculated on the basis of both 21

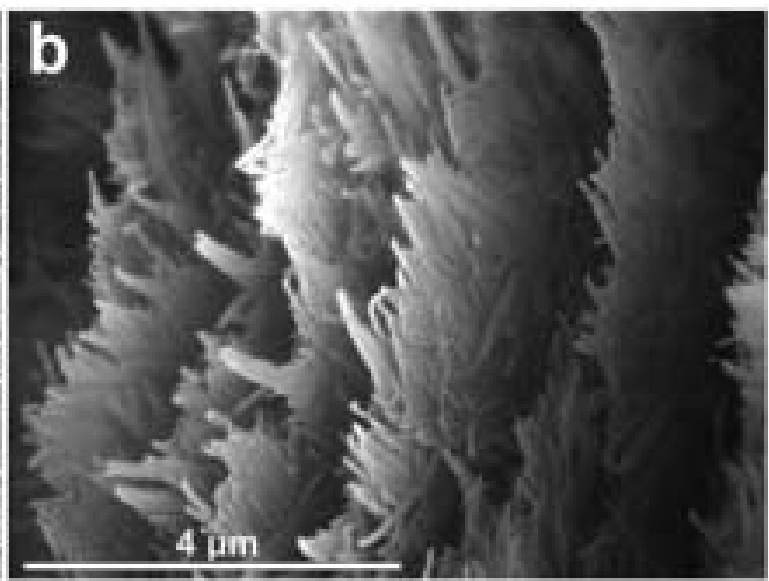

Figure 4. SEM images f the tightened growth of sepi lite-paly gerskite fibers formin planes. All fibers are of similar appearance and it is not pessible to distinguish sepi lite from palygersite. (a) Image of a plane $\bullet$ fibers. The axes f f the fibers arep intingin all directions and the aggregates form well defined planes. (b) Image of several contigueus planes $\bullet$ fibers. The $\bullet$ rientations $\bullet$ the fibers forming the parallellamination is clear. 


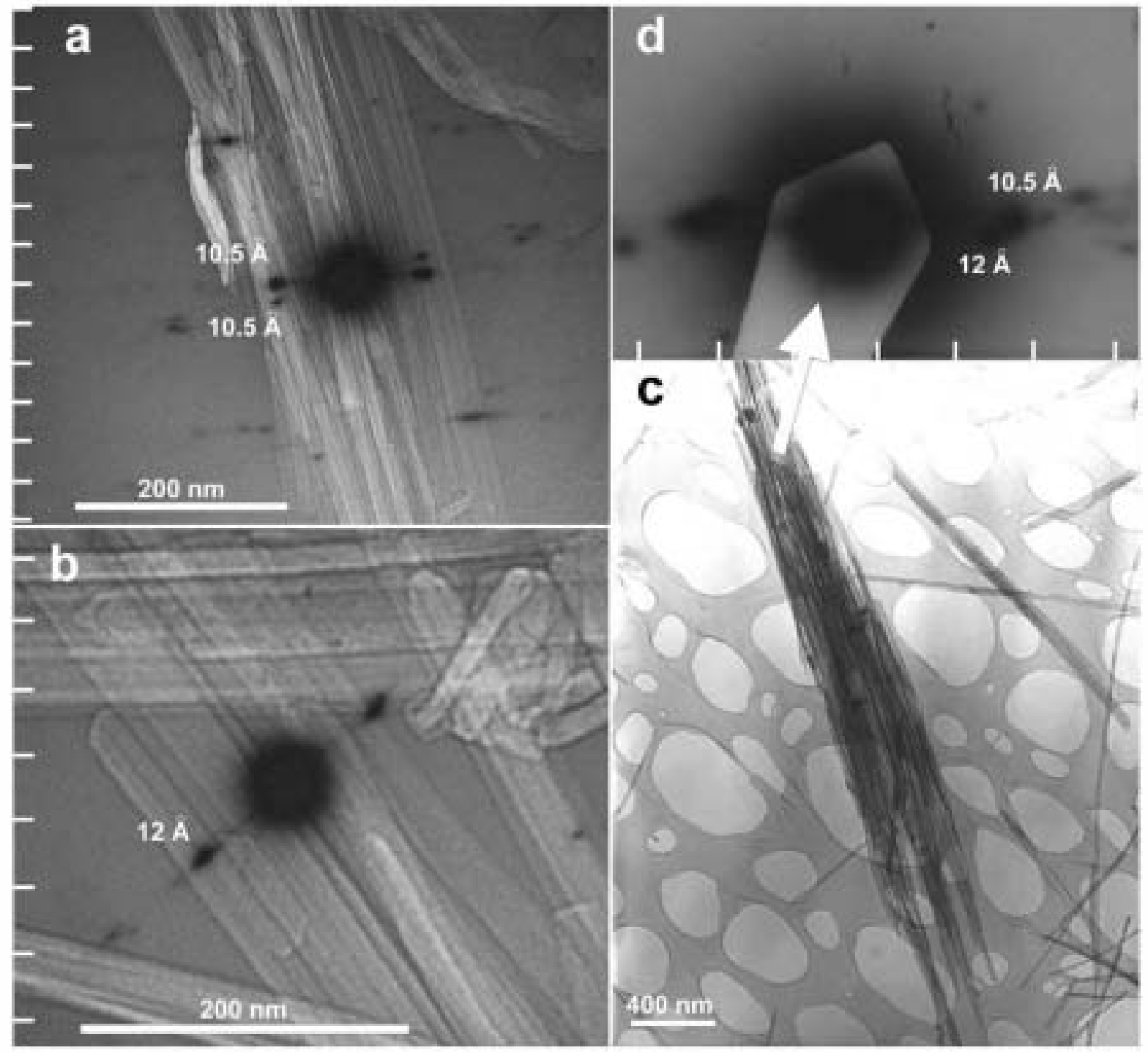

Figure 5. TEM images. (a,b) Superimpesed negatives (TEM image and SAED spets) on the image. Camera length (L) - 806 mm, $\lambda-0.25 \mathrm{~nm}$. Camera constant (L $\lambda)-20.1$ in all SAED images. There are tw $\bullet$ raphic scales: the vertical scale corresp $\bullet$ ds tothe SAED image ( $1 \mathrm{~mm}$ between bars) an the horizontal scale correspends to the TEM image. (a) There are tw 110 electron iffraction spots correspending to tw different bundles of fibers (10.5 $\dot{A}$ ) of paly

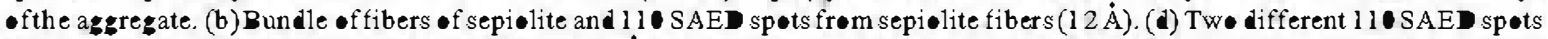
from sepi lite and palygorskite fibers (10.5 and $12 \dot{A}$ ) corresponding to tw contigu fibers that form a small angle in a same bunde. Scale: 1 mm between bars. (c) Bundles of sepi lite paly gerskite.

(Table 2) and 32 exygens per half unit-cell (as palygorskite and sepiolite, respectively) (Table 3) with the aim of separating two groups of analyses (these which fit well either as palygorskite or sepiolite) because it is not possible to identify the tw॰ minerals frem their morpholøgical features aløne. The results obtained show that most of the analyses do not correspond to either pure sepielite or pure palygorskite. There is no gap between the two groups of formulae, but on the contrary, both groups display a continuous variation. Whether the formulae are fitted as palygorskite or as sepiolite the results are continuous. If palygorskite composition is taken inte account (Table 2) there is only a small group - $f$ analyses which fit well as palygorskite. Most of the analyses fit with $\mathrm{M}$-rich palygorskite, and others are s• anomalous that they cannot be considered as palygorskite. However, if sepiolite composition is considered (Table 3) there is no analysis that fits as sepiolite and all analyses should correspend to a very anemaleus sepiolite with a very large proportion of octahedral $\mathrm{Al}$. There are tw॰ possibilities: (1) there is n॰ pure sepiolite and all analyses are a mixture of sepiolite and palygorskite; or (2) the sepielites are an an॰maløus Alrich sepiolite. 
Table 1. Chemical composition (wt.\%) of the isolated particles.

\begin{tabular}{|c|c|c|c|c|c|c|c|c|c|c|c|c|c|c|c|}
\hline & $\mathrm{Si}_{2}$ & $\mathrm{Al}_{2} \boldsymbol{\bigcirc}_{3}$ & $\mathrm{Mg} \bullet$ & $\mathrm{Fe}_{2} \boldsymbol{\Theta}_{3}$ & $\mathrm{Ti}_{2}$ & $\mathrm{Ca}$ & $\mathrm{K}_{2} \bullet$ & & $\mathrm{SiO}_{2}$ & $\mathrm{Al}_{2} \bullet_{3}$ & $\mathrm{Mg}$ & $\mathrm{Fe}_{2} \boldsymbol{\Theta}_{3}$ & $\mathrm{Ti}_{2}$ & $\mathrm{Ca}$ & $\mathrm{K}_{2}$ \\
\hline 1 & 70.19 & 4.07 & 22.60 & 2.77 & & •. 36 & & 51 & 70.38 & 10.77 & 16.80 & 2.00 & & & 0.60 \\
\hline 2 & 70.30 & 3.48 & 23.34 & 2.51 & & 0.35 & & 52 & 63.75 & 11.34 & 17.91 & 2.86 & 1.00 & 1.54 & 1.45 \\
\hline 3 & 69.46 & 6.26 & 20.39 & 3.25 & & 0.48 & & 53 & 69.74 & 7.75 & 19.73 & 2.00 & & 0.42 & 0.36 \\
\hline 4 & 70.66 & 8.80 & 14.98 & 5.19 & & & & 54 & 70.17 & 7.75 & 19.90 & 1.43 & $\bullet .33$ & 0.14 & 0.24 \\
\hline 5 & 67.88 & 9.71 & 17.30 & 4.39 & & 0.73 & & 55 & 67.60 & 10.39 & 16.41 & 5.00 & & & 0.48 \\
\hline 6 & 69.86 & 5.43 & 20.66 & 3.46 & & 0.48 & & 56 & 67.60 & 10.01 & 16.75 & 4.15 & & 0.7 & 0.84 \\
\hline 7 & 71.26 & 8.58 & 16.35 & 3.81 & & & & 57 & 61.83 & 10.58 & 20.06 & 3.29 & 4.17 & & \\
\hline 8 & 74.44 & 7.08 & 15.84 & 2.64 & & & & 58 & 64.82 & 3.17 & 19.07 & 2.29 & 3.17 & & 1.33 \\
\hline 9 & 73.28 & 5.94 & 17.64 & 3.12 & & & & 59 & 65.03 & 8.50 & 20.56 & 5.00 & 0.83 & & \\
\hline 10 & 76.28 & 8.97 & 15.35 & & & & & 60 & 66.10 & 6.99 & 23.05 & 3.86 & & & \\
\hline 11 & 71.14 & 4.29 & 22.26 & 2.31 & & & & 61 & 71.45 & 11.34 & 12.60 & 3.29 & & & 0.48 \\
\hline 12 & 72.26 & 10.59 & 13.95 & 3.21 & & & & 62 & 72.52 & 10.96 & 7.30 & 1.90 & $\bullet .33$ & $\bullet .56$ & 0.60 \\
\hline 13 & 69.73 & 13.45 & 13.61 & 3.21 & & & & 63 & 62.47 & 8.88 & 17.58 & 0.37 & 5.00 & 1.68 & \\
\hline 14 & 70.74 & 12.08 & 14.23 & 2.94 & & & & 64 & 70.60 & 9.64 & 19.07 & 0.71 & & & \\
\hline 15 & 71.27 & 11.17 & 13.97 & 3.59 & & & & 65 & 68.24 & 11.71 & 14.43 & 5.58 & & & \\
\hline 16 & 72.23 & 10.10 & 15.25 & 2.43 & & & & 66 & 71.45 & 10.58 & 13.26 & 4.00 & e. 67 & & \\
\hline 17 & 71.45 & 11.73 & 13.58 & 3.23 & & & & 67 & 66.75 & 12.09 & 11.20 & 2.72 & & & \\
\hline 18 & 71.03 & 11.89 & 13.88 & 3.20 & & & & 68 & 65.68 & 13.04 & 19.90 & 1.43 & & & \\
\hline 19 & 71.15 & 10.74 & 15.05 & 3.05 & & & & 69 & 68.89 & 5.29 & 24.21 & 1.57 & & & \\
\hline 20 & 71.66 & 7.52 & 17.77 & 2.89 & & •.17 & & 70 & 68.24 & 4.91 & 24.71 & 1.00 & $\bullet .33$ & $\bullet .07$ & 0.04 \\
\hline 21 & 70.71 & 6.24 & 19.44 & 2.83 & & 0.78 & & 71 & 65.68 & 4.53 & 25.87 & 3.00 & 0.33 & 0.56 & \\
\hline 22 & 72.15 & 6.57 & 15.96 & & & 3.51 & & 72 & 66.96 & 4.72 & 25.53 & 2.00 & & 0.84 & \\
\hline 23 & 72.71 & 8.66 & 11.05 & 6.68 & & & & 73 & 67.60 & 6.61 & 21.55 & 3.29 & 0.50 & 0.28 & 0.36 \\
\hline 24 & 72.05 & 4.05 & 19.25 & 2.56 & & 0.18 & 1.91 & 74 & 65.03 & 6.24 & 24.54 & 3.00 & 1.00 & & \\
\hline 25 & 73.23 & 5.39 & 17.51 & 3.62 & & & & 75 & 67.17 & 7.56 & 22.22 & 1.14 & 1.00 & 0.28 & 0.72 \\
\hline 26 & 69.76 & 5.09 & 16.46 & 2.76 & & & 5.6 & 76 & 60.97 & 8.12 & 25.53 & 1.29 & 2.17 & 0.84 & 1.08 \\
\hline 27 & 70.3 & 6.65 & 18.16 & 2.75 & & & 1.96 & 77 & 69.74 & $8.5 \bullet$ & 16.91 & 2.00 & & 0.98 & 1.93 \\
\hline 28 & 68.75 & 4.78 & 22.7 & 2.18 & & & 1.49 & 78 & 57.12 & 10.20 & 25.87 & 4.72 & & 1.26 & 0.84 \\
\hline 29 & 73.46 & 8.48 & 14.95 & 2.64 & & 0. 47 & & 79 & 71.88 & 9.64 & 2.82 & $\bullet .14$ & & & \\
\hline 30 & 71.59 & 8.68 & 15.63 & 3.52 & & & & 80 & 65.68 & 10.96 & 21.22 & 1.14 & & 0.10 & $\bullet .13$ \\
\hline 31 & 70.42 & 13.68 & 11.09 & 3.94 & & & & 81 & 69.10 & 10.96 & 16.75 & 2.72 & & 0.08 & \\
\hline 32 & 72.08 & 6.71 & 21.21 & & & & & 82 & 66.96 & 10.58 & 16.75 & 2.90 & & 1.26 & 0.36 \\
\hline 33 & 70.18 & 10.18 & 19.64 & & & & & 83 & 67.17 & 11.71 & 17.91 & 1.43 & 1.17 & 0.28 & •. 36 \\
\hline 34 & 73.46 & 8.31 & 18.23 & & & & & 84 & 65.25 & 11.71 & 18.90 & 1.57 & 1.83 & 0.13 & \\
\hline 35 & 69.11 & 5.33 & 20.66 & 3.75 & & & 0.81 & 85 & 56.26 & 13.04 & 20.73 & 5.15 & 2.17 & 0.98 & 1.81 \\
\hline 36 & 71.18 & 10.00 & 13.16 & 5.12 & & 0.53 & & 86 & 56.05 & 11.90 & 21.22 & 4.86 & 3.17 & 1.54 & 1.20 \\
\hline 37 & 68.69 & 4.98 & 23.53 & 2.05 & & $\bullet .16$ & 0.55 & 87 & 71.24 & 10.01 & 13.10 & 4.15 & 1.00 & 0.42 & $\bullet .36$ \\
\hline 38 & 69.49 & 7.41 & 18.63 & 3.93 & & & & 88 & 72.52 & 8.69 & 12.27 & 4.00 & 1.67 & 0.28 & 0.48 \\
\hline 39 & 69.53 & 5.85 & 21.23 & 2.69 & & 0.70 & & 89 & 57.33 & 9.45 & 25.04 & & 1.67 & 2.52 & 3.85 \\
\hline 40 & 73.73 & 4.32 & 19.98 & 1.97 & & & & 90 & 59.04 & 9.26 & 23.88 & & & 3.50 & 4.46 \\
\hline 41 & 70.22 & 6.00 & 21.27 & 2.51 & & & & 91 & 66.10 & 3.97 & 27.19 & 2.43 & & & 0.60 \\
\hline 42 & $7 \bullet .37$ & 4.89 & 21.81 & 2.93 & & & & 92 & 71.45 & 7.94 & 17.08 & 2.57 & 0.33 & 0.42 & \\
\hline 43 & 69.72 & 5.45 & 22.8 & 1.96 & & & & 93 & 72.59 & 8.12 & 17.24 & 1.72 & & & \\
\hline 44 & 69.02 & 5.82 & 21.44 & 3.37 & & & & 94 & 55.41 & 5.67 & 19.57 & 3.86 & & 3.92 & 3.73 \\
\hline 45 & 66.32 & 7.18 & 19.90 & 3.15 & 1.83 & 0.98 & 0.84 & 95 & 61.83 & 6.05 & 21.89 & & 4.00 & 2.66 & 3.37 \\
\hline 46 & 68.89 & 7.75 & 21.72 & 1.72 & & & & 96 & 72.52 & $8.5 \bullet$ & 15.25 & 3.15 & •.67 & & \\
\hline 47 & 59.69 & 6.05 & 25.53 & 8.86 & & & & 97 & 69.53 & 9.26 & 15.92 & 3.43 & 0.83 & 0.42 & 0.60 \\
\hline 48 & 63.32 & 6.42 & 24.21 & 6.00 & & & & 98 & 67.40 & 5.48 & 21.55 & 1.86 & 2.50 & 0.98 & 0.36 \\
\hline 49 & 66.10 & 10.58 & 18.90 & 4.43 & & & & 99 & 70.38 & 6.80 & 17.91 & 3.00 & 0.83 & 0.84 & 0.24 \\
\hline 50 & 65.80 & $8.5 \bullet$ & 18.90 & 5.15 & & 0.84 & 0.72 & & & & & & & & \\
\hline
\end{tabular}

\section{DISCUSSION AND CONCLUSIONS}

Sepielite is a $\mathrm{Mg}$ silicate with negligible isømerphic substitution that has eight pøssible •ctahedral pøsitions per half unit-cell, all of them occupied by $\mathrm{Mg}$. According to Newman and Brown (1987) the total munber of •ctahedral cations ranges from 7.01 t॰ 8.01 , and is clese to 8 according to Galán and Carreter (1999) whe confirmed that bøth tetrahedral and ectahedral substitutions are negligible and cannot be detected by the EXX technique. Nevertheless, if the structural formulae from all of the Allou Kagne analyses are calculated as sepiolite, the number of $\bullet$ ctahedral cations ranges from 3.12 and 8.04 and the ectahedral $\mathrm{Mg}$ number varies frøm 1.73 to 7.09 (4.81 $\bullet$ n average) (Table 3), which means that mest analyses have a number $\bullet$ octahedral catiøns and $\mathrm{Mg}$ values which are 
Table 2. Crystallo-chemical formulae calculated on basis of 21 oxygens per half unit-cell (as palygorskite). Formulae have been ordered according to their content of octahedral cations.

\begin{tabular}{|c|c|c|c|c|c|c|c|c|c|c|c|c|c|c|c|c|c|c|c|}
\hline & $\mathrm{Si}$ & ${ }^{\mathrm{IV}} \mathrm{Al}$ & ${ }^{\mathrm{VI}} \mathrm{Al}$ & $\mathrm{Fe}^{3+}$ & $\mathrm{Mg}$ & $\mathrm{Ti}$ & $\Sigma O$ & $\mathrm{Ca}$ & $\mathrm{K}$ & & $\mathrm{Si}$ & ${ }^{\mathrm{IV}} \mathrm{Al}$ & ${ }^{\mathrm{VI}} \mathrm{Al}$ & $\mathrm{Fe}^{3+}$ & $\mathrm{Mg}$ & $\mathrm{Ti}$ & $\Sigma O$ & $\mathrm{Ca}$ & $\mathrm{K}$ \\
\hline 11 & 8.05 & & 0.29 & $\bullet .10$ & 1.88 & & 2.27 & & & 55 & 7.72 & 0.28 & 1.12 & ๑.43 & 2.79 & & 4.34 & & 0.07 \\
\hline 22 & 8.17 & & •.88 & & 2.69 & & 3.57 & 0.43 & & 52 & 7.37 & 0.63 & 0.92 & 0.25 & 3.09 & 0.09 & 4.35 & 0.19 & 0.21 \\
\hline 36 & 8.04 & & 1.33 & 0.04 & 2.21 & & 3.58 & 0.06 & & 5 & 7.73 & 0.27 & 1.03 & 0.38 & 2.94 & & 4.35 & 0.09 & \\
\hline 23 & 8.22 & & 1.15 & 0.57 & 1.86 & & 3.58 & & & 21 & 8.01 & & ๑.83 & 0.24 & 3.28 & & 4.35 & 0.09 & \\
\hline 29 & 8.35 & & 0.98 & 0.26 & $2.4 \bullet$ & & 3.64 & 0.02 & & 38 & 7.89 & $\mathbf{0 . 1 1}$ & •. 88 & $\bullet .34$ & 3.15 & & 4.37 & & \\
\hline 10 & 8.37 & & 1.16 & & 2.51 & & 3.67 & & & 53 & 7.89 & $\mathbf{0 . 1 1}$ & 0.92 & 0.17 & 3.33 & & 4.42 & 0.05 & 0.05 \\
\hline 88 & 8.17 & & 1.15 & 0.34 & 2.06 & 0.14 & 3.69 & 0.03 & 0.07 & 64 & 7.91 & 0.09 & 1.18 & 0.06 & 3.18 & & 4.42 & & \\
\hline 54 & 7.92 & 0.08 & 0.23 & 0.12 & 3.35 & 0.03 & 3.73 & 0.02 & 0.03 & 32 & 8.07 & & 0.88 & & 3.54 & & 4.42 & & \\
\hline 62 & 8.14 & & 1.45 & 0.23 & 2.03 & 0.03 & 3.74 & 0.07 & 0.09 & 45 & 7.62 & 0.38 & 0.59 & 0.27 & 3.41 & •.16 & 4.43 & 0.12 & 0.12 \\
\hline 26 & 8.05 & & 0.69 & 0.24 & 2.83 & & 3.76 & & 1.27 & 58 & 7.43 & 0.57 & $\bullet .73$ & 0.20 & 3.26 & 0.27 & 4.46 & & 0.19 \\
\hline 8 & 8.32 & & 0.93 & 0.22 & 2.64 & & 3.79 & & & 50 & 7.60 & 0.40 & $\bullet .76$ & 0.45 & 3.25 & & 4.46 & $0.1 \bullet$ & •.11 \\
\hline 61 & 8.04 & & 1.50 & 0.28 & 2.11 & & 3.89 & & 0.07 & 3 & 7.89 & $\boldsymbol{0} .11$ & $\bullet .73$ & 0.28 & 3.45 & & 4.46 & 0.06 & \\
\hline 31 & 7.91 & 0.09 & 1.72 & $\bullet .33$ & 1.86 & & 3.91 & & & 35 & 7.90 & $\mathbf{0 . 1 \bullet}$ & $\bullet .62$ & 0.32 & 3.52 & & 4.46 & & ๑.18 \\
\hline 87 & 8.02 & & 1.33 & $\bullet .35$ & 2.20 & 0.08 & 3.96 & 0.05 & 0.05 & 33 & 7.85 & 0.15 & 1.19 & & 3.28 & & 4.47 & & \\
\hline 25 & 8.25 & & 0.72 & 0.31 & 2.94 & & 3.97 & & & 6 & 7.94 & 0.06 & 0.67 & 0.30 & 3.50 & & 4.47 & 0.06 & \\
\hline 95 & 7.29 & $\mathbf{0 . 7 1}$ & $\bullet .13$ & & 3.85 & & 3.98 & 0.34 & 0.51 & 39 & 7.90 & $0.1 \bullet$ & $\bullet .68$ & 0.23 & 3.59 & & 4.50 & 0.09 & \\
\hline 12 & 8.09 & & 1.40 & 0.27 & 2.33 & & 4.00 & & & 67 & 7.55 & 0.45 & 1.16 & 0.23 & 3.13 & & 4.52 & & \\
\hline 96 & 8.13 & & 1.12 & 0.27 & 2.55 & 0.06 & 4.00 & & & 49 & 7.54 & 0.46 & $\bullet .96$ & $\bullet .38$ & 3.21 & & 4.55 & & \\
\hline 9 & 8.23 & & 0.79 & 0.26 & 2.95 & & 4.00 & & & 41 & 7.95 & 0.05 & $\bullet .75$ & 0.21 & 3.59 & & 4.55 & & \\
\hline 66 & 8.03 & & 1.40 & 0.34 & 2.22 & 0.06 & 4.02 & & & 90 & 7.02 & 0.98 & $\bullet .32$ & & 4.23 & & 4.55 & 0.45 & 0.68 \\
\hline 24 & 8.18 & & 0.54 & 0.22 & 3.26 & & 4.02 & 0.02 & 0.42 & 28 & 7.86 & - .14 & $\bullet .50$ & 0.19 & 3.87 & & 4.56 & & 0.33 \\
\hline 30 & 8.06 & & 1.15 & 0.30 & 2.62 & & 4.07 & & & 85 & 6.65 & 1.35 & 0.47 & 0.46 & 3.65 & & 4.58 & 0.12 & 0.27 \\
\hline 16 & 8.08 & & 1.33 & 0.20 & 2.54 & & 4.07 & & & 73 & 7.71 & 0.29 & $\bullet .60$ & 0.28 & 3.67 & 0.04 & 4.59 & 0.03 & 0.05 \\
\hline 93 & 8.15 & & 1.07 & •. 14 & 2.87 & & 4.08 & & & 42 & 7.98 & 0.02 & $\bullet .65$ & 0.25 & 3.69 & & 4.59 & & \\
\hline 40 & 8.28 & & 0.57 & 0.17 & 3.34 & & 4.08 & & & 44 & 7.86 & $\mathbf{0} .14$ & 0.64 & 0.32 & 3.64 & & 4.60 & & \\
\hline 17 & 8.00 & & 1.55 & 0.27 & 2.27 & & 4.09 & & & 1 & 7.98 & 0.02 & $\bullet .53$ & 0.24 & 3.83 & & 4.60 & 0.04 & \\
\hline 34 & 8.17 & & 1.09 & & 3.02 & & 4.11 & & & 75 & 7.65 & 0.35 & $\bullet .66$ & $\bullet .1 \bullet$ & 3.77 & 0.09 & 4.62 & 0.03 & 0.11 \\
\hline 18 & 7.96 & 0.04 & 1.53 & 0.27 & 2.32 & & 4.12 & & & 2 & 7.99 & 0.01 & $\bullet .46$ & 0.21 & 3.95 & & 4.62 & 0.04 & \\
\hline 15 & 8.00 & & 1.48 & 0.30 & 2.34 & & 4.12 & & & 46 & 7.79 & 0.21 & $\bullet .82$ & 0.15 & 3.66 & & 4.63 & & \\
\hline 77 & 7.95 & 0.05 & 1.09 & 0.17 & 2.87 & & 4.13 & 0.12 & 0.28 & 68 & 7.43 & 0.57 & 1.17 & 0.12 & 3.35 & & 4.64 & & \\
\hline 4 & 8.00 & 0.06 & 1.17 & 0.44 & 2.53 & & 4.14 & & & 80 & 7.47 & 0.53 & 0.94 & $\bullet .1 \bullet$ & 3.60 & & 4.64 & 0.07 & 0.09 \\
\hline 13 & 7.82 & •.18 & 1.60 & 0.27 & 2.28 & & 4.15 & & & 59 & 7.48 & $\bullet .52$ & 0.63 & $\bullet .43$ & 3.52 & 0.07 & 4.65 & & \\
\hline 14 & 7.93 & 0.07 & 1.53 & 0.25 & 2.38 & & 4.16 & & & 43 & 7.90 & $\mathbf{0 . 1 \bullet}$ & $\bullet .63$ & 0.17 & 3.86 & & 4.66 & & \\
\hline 92 & 8.06 & & 1.05 & 0.22 & 2.87 & 0.03 & 4.17 & 0.05 & & 57 & 7.12 & 0.88 & $\bullet .56$ & 0.29 & 3.45 & 0.36 & 4.66 & & \\
\hline 97 & 7.89 & - .11 & 1.13 & 0.29 & 2.69 & 0.07 & 4.18 & 0.05 & 0.09 & 98 & $7.7 \bullet$ & 0.30 & •. 44 & •.16 & 3.86 & 0.22 & 4.68 & $\mathbf{0 . 1 2}$ & 0.05 \\
\hline 19 & 7.98 & 0.02 & 1.40 & 0.26 & 2.52 & & 4.18 & & & 37 & 7.83 & 0.17 & $\bullet .50$ & •.18 & 4.00 & & 4.68 & 0.02 & 0.12 \\
\hline 27 & 8.00 & & 0.89 & 0.24 & 3.08 & & 4.21 & & $\bullet .43$ & 70 & 7.72 & 0.28 & $\bullet .38$ & 0.09 & 4.19 & 0.03 & 4.69 & 0.07 & 0.04 \\
\hline 7 & 8.03 & & 1.14 & 0.32 & 2.75 & & 4.21 & & & 86 & 6.63 & 1.37 & 0.29 & 0.43 & 3.74 & •.28 & 4.74 & 0.20 & 0.18 \\
\hline 20 & 8.07 & & 1.00 & •. 24 & 2.98 & & 4.22 & 0.02 & & 89 & 6.83 & 1.17 & •.16 & & 4.44 & 0.15 & 4.75 & 0.32 & 0.59 \\
\hline 51 & 7.93 & 0.07 & 1.36 & e.17 & $2.7 \bullet$ & & 4.23 & & 0.09 & 69 & 7.82 & 0.18 & $\bullet .53$ & •.13 & 4.10 & & 4.76 & & \\
\hline 79 & 8.03 & & 1.27 & •.14 & 2.82 & & 4.23 & & & 60 & 7.57 & •.43 & $\bullet .51$ & $\bullet .33$ & 3.94 & & 4.78 & & \\
\hline 65 & 7.75 & 0.25 & 1.32 & 0.48 & 2.44 & & 4.24 & & & 72 & 7.66 & 0.34 & $\bullet .30$ & 0.17 & 4.36 & & 4.83 & $0.1 \bullet$ & \\
\hline 99 & 7.98 & 0.02 & 0.89 & 0.26 & 3.03 & 0.07 & 4.25 & $\mathbf{0 . 1 \bullet}$ & 0.03 & 74 & 7.48 & $\boldsymbol{0 . 5 2}$ & $\bullet .33$ & $\bullet .26$ & 4.21 & 0.09 & 4.89 & & \\
\hline 82 & 7.65 & $\bullet .35$ & 1.07 & $\bullet .36$ & 2.85 & & 4.28 & •. 15 & 0.05 & 71 & 7.56 & 0.44 & ๑.18 & $\bullet .26$ & 4.44 & 0.03 & 4.91 & 0.07 & \\
\hline 56 & 7.72 & •. 28 & 1.07 & 0.36 & 2.85 & & 4.28 & 0.09 & 0.12 & 76 & 7.09 & 0.91 & 0.20 & $\mathbf{0 . 1 1}$ & 4.42 & 0.19 & 4.92 & $0.1 \bullet$ & e.16 \\
\hline 94 & 7.23 & 0.77 & 0.10 & 0.38 & 3.81 & & 4.29 & $\bullet .55$ & $\bullet .62$ & 48 & 7.35 & 0.65 & 0.23 & 0.52 & 4.19 & & 4.94 & & \\
\hline 83 & 7.60 & $\bullet .40$ & 1.16 & 0.12 & 3.02 & & 4.30 & 0.03 & 0.05 & 91 & 7.59 & ๑.41 & $\mathbf{0 . 1 3}$ & 0.21 & 4.65 & & 4.99 & & 0.09 \\
\hline 81 & 7.80 & 0.20 & 1.26 & 0.23 & 2.82 & & 4.31 & 0.05 & & 78 & 6.74 & 1.26 & •.16 & 0.42 & 4.55 & & 5.13 & 0.16 & 0.13 \\
\hline 63 & 7.25 & 0.75 & $\bullet .46$ & 0.37 & 3.04 & 0.44 & 4.31 & $\boldsymbol{0 . 2 1}$ & & 47 & 7.04 & •.84 & & 0.79 & 4.49 & & 5.28 & & \\
\hline 84 & 7.42 & 0.58 & 0.99 & 0.13 & 3.20 & & 4.32 & 0.09 & & & & & & & & & & & \\
\hline
\end{tabular}

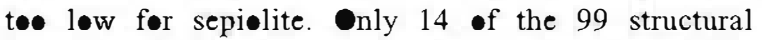
formulae ebtained as sepiølite have $>6$ ectahedral $\mathrm{Mg}$ atems per half unit-cell. Taking inte acceunt the values

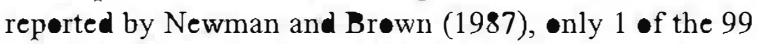
structural formulae calculated in this work could be considered unequivecally as sepielite. Furthermere, this sepielite wøuld have a vacant pesition per half unit-cell.

On the •ther hand, palygorskite can display greater cœmpesitiønal variations than sepiølite. Galán and
Carreter (1999) confirmed that palygorskite contains mainly $\mathrm{Mg}, \mathrm{Al}$ and $\mathrm{Fe}$ with an $\boldsymbol{R} 2 / \boldsymbol{R} 3$ rativ clese to 1 . On average, four of every five octahedral palygorskite positions are occupied. According to Newman and Brøwn (1987), the sum of •ctahedral cations lies between 3.76 and 4.64 , with a mean value of 4.00 . García Rømer et al. (2004) repørted a very Mg-rich palygerskite that has 4.36 octahedral catiens per half unit-cell. They studied a very large number of samples 
Table 3. Crystallo-chemical formulae calculated on the basis of 32 oxygens per half unit-cell (as sepiolite). The formulae have been ordered according to their $\mathrm{Mg}$ content.

\begin{tabular}{|c|c|c|c|c|c|c|c|c|c|c|c|c|c|c|c|c|c|c|c|}
\hline & $\mathrm{Si}$ & ${ }^{\mathrm{IV}} \mathrm{Al}$ & ${ }^{\mathrm{VI}} \mathrm{Al}$ & $\mathrm{Fe}^{3+}$ & $\mathrm{Mg}$ & $\mathrm{Ti}$ & $\Sigma 0$ & $\mathrm{Ca}$ & $\mathrm{K}$ & & $\mathrm{Si}$ & ${ }^{\mathrm{IV}} \mathrm{Al}$ & ${ }^{\mathrm{VI}} \mathrm{Al}$ & $\mathrm{Fe}^{3+}$ & $\mathrm{Mg}$ & $\mathrm{Ti}$ & $\Sigma O$ & $\mathrm{Ca}$ & $\mathrm{K}$ \\
\hline 17 & 12.19 & & 1.18 & 0.21 & 1.73 & & 3.12 & & & 49 & 11.49 & 0.51 & 1.66 & 0.58 & 4.90 & & 7.14 & & \\
\hline 13 & 11.92 & 0.08 & 1.24 & 0.21 & 1.73 & & 3.18 & & & 50 & 11.58 & 0.42 & 1.34 & 0.68 & 4.95 & & 6.97 & e. 16 & อ. 16 \\
\hline 15 & 12.18 & & 1.13 & 0.23 & 1.78 & & 3.14 & & & 58 & 11.32 & 0.68 & 1.30 & $\bullet .3 \bullet$ & 4.96 & 0.42 & 6.98 & & 0.30 \\
\hline 19 & 12.16 & & 1.08 & 0.20 & 1.92 & & 3.20 & & & 24 & 12.47 & & 0.83 & 0.33 & 4.97 & & 6.13 & 0.03 & 0.64 \\
\hline 31 & 12.05 & & 2.76 & 0.51 & 2.83 & & 6.10 & & & 33 & 11.97 & 0.03 & 2.02 & & 4.99 & & 7.01 & & \\
\hline 23 & 12.53 & & 1.76 & 0.87 & 2.84 & & 5.47 & & & 21 & 12.20 & & 1.27 & 0.37 & 5.00 & & 6.64 & •. 14 & \\
\hline 62 & 12.41 & & 2.21 & 0.35 & 3.09 & 0.04 & 5.69 & $0.1 \bullet$ & 0.13 & 53 & 12.03 & & 1.57 & 0.26 & 5.07 & & 6.90 & 0.08 & 0.08 \\
\hline 88 & 12.45 & & 1.76 & 0.52 & 3.14 & 0.22 & 5.64 & 0.05 & 0.11 & 40 & 12.61 & & 0.87 & 0.25 & 5.09 & & 6.21 & & \\
\hline 61 & 12.25 & & 2.29 & 0.42 & 3.22 & & 5.93 & •.15 & 0.11 & 54 & 12.07 & & 1.57 & •. 18 & $5.1 \bullet$ & 0.04 & 6.89 & 0.03 & 0.05 \\
\hline 87 & 12.22 & & 2.02 & 0.54 & 3.35 & 0.13 & 6.04 & 0.08 & 0.08 & 68 & 11.32 & •.68 & 1.97 & 0.19 & 5.11 & & 7.27 & & \\
\hline 36 & 12.25 & & 2.03 & 0.66 & 3.37 & & 6.06 & $\bullet .1 \bullet$ & & 45 & 11.60 & 0.40 & 1.08 & •.41 & 5.19 & 0.24 & 6.92 & 0.18 & 0.19 \\
\hline 66 & 12.23 & & 2.14 & 0.52 & 3.39 & 0.09 & 6.14 & & & 57 & 10.86 & 1.14 & 1.05 & 0.43 & 5.25 & 0.21 & 6.94 & & \\
\hline 18 & 12.13 & & 2.39 & 0.41 & 3.53 & & 6.33 & & & 3 & 12.03 & & 1.28 & 0.42 & 5.26 & & 6.96 & 0.09 & \\
\hline 12 & 12.32 & & 2.13 & 0.41 & 3.55 & & 6.09 & & & 6 & 12.11 & & 1.11 & 0.45 & 5.33 & & 6.89 & 0.09 & \\
\hline 14 & 12.08 & & 2.43 & 0.38 & 3.62 & & 6.43 & & & 35 & 12.03 & & 1.09 & 0.49 & 5.36 & & 6.94 & & 0.27 \\
\hline 65 & 11.80 & 0.2 & 2.19 & 0.73 & 3.72 & & 6.64 & & & 59 & 11.39 & 0.61 & 1.15 & •.66 & 5.37 & •.11 & 7.29 & & \\
\hline 29 & 12.53 & & $1.7 \bullet$ & 0.34 & 3.80 & & 5.84 & 0.09 & & 32 & 12.29 & & 1.35 & & 5.39 & & 6.74 & & \\
\hline 10 & 12.81 & & 1.77 & & 3.84 & & 5.61 & & & 41 & 12.11 & & 1.22 & $\bullet .33$ & 5.46 & & 7.1 & & \\
\hline 4 & 12.19 & & 1.79 & 0.67 & 3.85 & & 6.31 & & & 39 & 12.04 & & 1.19 & 0.35 & 5.48 & & 7.02 & 0.13 & \\
\hline 16 & 12.31 & & 2.03 & 0.31 & 3.87 & & 6.21 & & & 80 & 11.38 & 0.62 & 1.62 & •. 15 & 5.48 & & 7.25 & 0.10 & •.13 \\
\hline 96 & 12.39 & & 1.71 & 0.40 & 3.88 & 0.09 & 6.08 & & & 44 & 11.97 & 0.03 & 1.16 & 0.49 & 5.54 & & 7.19 & & \\
\hline 30 & 12.29 & & 1.76 & 0.45 & 4.00 & & 6.21 & & & 85 & 10.13 & 1.87 & 0.90 & 0.70 & 5.56 & 0.29 & 7.45 & 0.19 & 0.41 \\
\hline 8 & 12.67 & & 1.42 & 0.34 & 4.02 & & 5.78 & & & 46 & 11.87 & 0.13 & 1.44 & 0.22 & 5.58 & & 7.24 & & \\
\hline 22 & 12.45 & & 1.34 & & $4.1 \bullet$ & & 5.44 & $\bullet .65$ & & 73 & 11.76 & 0.24 & 1.12 & 0.43 & 5.59 & 0.07 & 7.21 & 0.05 & 0.08 \\
\hline 97 & 12.02 & & 1.89 & 0.45 & 4.10 & 0.11 & 6.55 & 0.08 & •.13 & 98 & 11.73 & 0.27 & 0.86 & 0.24 & 5.61 & 0.33 & 7.04 & 0.18 & 0.08 \\
\hline 51 & 12.08 & & 2.18 & 0.26 & 4.11 & & 6.55 & & •. 13 & 42 & 12.16 & & 1.00 & •. 38 & 5.62 & & 7.00 & & \\
\hline 7 & 12.24 & & 1.74 & 0.49 & 4.19 & & 6.42 & & & 86 & 10.11 & 1.89 & 0.64 & $\bullet .66$ & $5.7 \bullet$ & •.43 & 7.43 & 0.30 & 0.28 \\
\hline 55 & 11.76 & 0.24 & 1.89 & 0.65 & 4.26 & & 6.80 & & 0.11 & 11 & 12.26 & & 0.87 & $\bullet .3 \bullet$ & 5.72 & & 6.89 & & \\
\hline 79 & 12.24 & & 1.24 & 0.22 & 4.29 & & 5.75 & & & 75 & 11.66 & 0.34 & 1.21 & •. 15 & 5.75 & $\bullet .13$ & 7.24 & 0.50 & อ. 16 \\
\hline 81 & 11.88 & 0.22 & 2.00 & 0.35 & 4.29 & & 6.64 & 0.08 & & 94 & 11.00 & 1.00 & 1.33 & 0.58 & 5.80 & & 7.71 & $\bullet .83$ & 0.95 \\
\hline 26 & 12.27 & & 1.05 & 0.37 & 4.31 & & 5.73 & & 1.94 & 1 & 12.16 & & 0.83 & $\bullet .36$ & 5.83 & & 7.02 & 0.07 & \\
\hline 82 & 11.65 & 0.35 & 1.82 & 0.54 & 4.34 & & 6.70 & 0.23 & 0.08 & 95 & 11.12 & 0.88 & 0.40 & & 5.87 & $\bullet .54$ & 6.81 & 0.51 & 0.77 \\
\hline 56 & 11.77 & 0.23 & 1.82 & 0.54 & 4.34 & & $6.7 \bullet$ & $\bullet .13$ & 0.19 & 43 & 12.04 & & 1.11 & 0.25 & 5.88 & & 7.24 & & \\
\hline 92 & 12.28 & & 1.61 & 0.33 & 4.37 & 0.04 & 6.35 & 0.08 & & 28 & 11.97 & 0.03 & 0.95 & 0.29 & 5.89 & & 7.13 & & 0.50 \\
\hline 93 & 12.42 & & 1.63 & 0.22 & 4.38 & & 6.23 & & & 60 & 11.54 & 0.46 & 0.98 & •. .51 & 6.00 & & 7.49 & & \\
\hline 77 & 12.11 & & 1.74 & 0.26 & 4.38 & & 6.38 & •.18 & 0.43 & & & & & & & & & & \\
\hline 5 & 11.78 & 0.22 & 1.77 & 0.57 & 4.47 & & 6.81 & ๑. 14 & & 2 & 12.18 & & $\mathbf{0 . 7 1}$ & $\bullet .33$ & 6.02 & & 7.06 & 0.07 & \\
\hline 25 & 12.57 & & 1.09 & 0.47 & 4.48 & & 6.04 & & & 37 & 11.92 & 0.08 & 0.94 & 0.27 & $6.1 \bullet$ & & 7.31 & 0.03 & อ. 18 \\
\hline 9 & 12.55 & & 1.20 & 0.40 & 4.50 & & $6.1 \bullet$ & & & 69 & 11.92 & 0.08 & 1.00 & 0.20 & 6.24 & & 7.44 & & \\
\hline 20 & 12.29 & & 1.52 & 0.37 & 4.54 & & 6.43 & 0.03 & & 48 & 11.20 & 0.80 & 0.54 & 0.80 & 6.38 & & 7.72 & & \\
\hline 83 & 11.58 & 0.42 & 1.96 & 0.19 & 4.60 & & 6.75 & 0.05 & 0.08 & 70 & 11.84 & 0.16 & ๑.84 & $\bullet .13$ & 6.39 & 0.04 & 7.40 & $0.1 \bullet$ & 0.05 \\
\hline 34 & 12.45 & & 1.66 & & 4.61 & & 6.27 & & & 74 & $11.4 \bullet$ & 0.60 & 0.69 & $\bullet .4 \bullet$ & 6.41 & ๑. 13 & 7.63 & & \\
\hline 99 & 12.16 & & 1.39 & 0.39 & 4.61 & ๑.11 & 6.50 & •.16 & 0.05 & 90 & $10.7 \bullet$ & 1.30 & 0.68 & & 6.45 & & 7.13 & 0.68 & 1.03 \\
\hline 63 & 11.04 & 0.96 & 0.89 & 0.57 & 4.63 & 0.67 & 6.76 & $\bullet .32$ & & 72 & 11.68 & 0.32 & $\bullet .65$ & •.26 & 6.64 & & 7.55 & •.16 & \\
\hline 27 & 12.18 & & 1.36 & 0.36 & 4.69 & & 6.41 & & 0.66 & 76 & 10.80 & 1.20 & 0.50 & 0.17 & 6.74 & 0.29 & $7.7 \bullet$ & 0.16 & 0.24 \\
\hline 52 & 11.24 & 0.76 & 1.60 & 0.38 & 4.70 & & 6.68 & 0.29 & $\bullet .33$ & 71 & 11.52 & 0.48 & ๑. 46 & 0.40 & 6.76 & 0.04 & 7.66 & 0.11 & \\
\hline 67 & 11.51 & 0.49 & 1.97 & 0.35 & 4.77 & & 7.09 & & & 89 & 10.40 & 1.60 & 0.42 & & 6.77 & 0.23 & 7.42 & 0.49 & 0.89 \\
\hline 38 & 12.03 & & 1.51 & 0.51 & 4.81 & & 6.83 & & & 47 & 10.72 & 1.28 & & 1.20 & 6.84 & & 8.04 & & \\
\hline 64 & 12.05 & & 1.94 & 0.09 & 4.85 & & 6.88 & & & 78 & 10.27 & 1.73 & •.43 & •. 64 & 6.93 & & 8.00 & 0.24 & 0.19 \\
\hline 84 & 11.31 & 0.69 & 1.70 & 0.21 & 4.88 & 0.24 & 7.03 & 0.13 & & 91 & 11.57 & 0.43 & 0.39 & 0.32 & 7.09 & & 7.80 & & 0.13 \\
\hline
\end{tabular}

from different lecalities and verified that in all cases the $\mathrm{Al}$ content is less than the $\mathrm{Mg}$ content in the ectahedral sheet, even theugh the rati $R^{3+}: \mathrm{Mg}$ is clese te 1 , due to the presence of $\mathrm{Fe}^{3+}$ in mest samples. In addition, the number $\bullet$ is morphic substitutions is considerable. The structural formula of this palygerskite has somewhat more $\mathrm{Mg}$ than $\mathrm{Al}$, with a rati $\bullet \mathrm{fg} / \mathrm{Al}$ close to 1.3 and a small proportion of $\mathrm{Fe}^{3+}$. Taking inte account the 99 structural formulae calculated on the basis of $21 \bullet x y$ gens per half unit-cell (as palygerskite), it is pessible t• verify that there are many structural formulae that can correspend te palygerskite but they shøw a very wide compesitional variation, ranging from terms very close to the ideal formula (Al/Mg clese to 1$)$ to others which are mere magnesic (Table 2).

Calculations of structural formulae shøuld give tw•

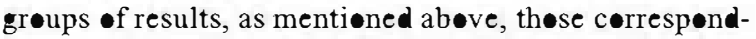
ing to palygerskite that will fit to 21 exygens per half 
unit-cell, and these corresponding to sepielite that will fit better to 32 exygens. Figures 7 and 8 contain all analyses fitted to both possibilities, palygorskite and

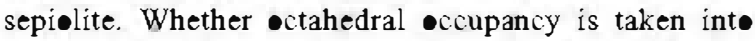
account (number and type of cations) or whether terahedral content is considered, the plots show a group of continueus points, and in ne case de the twe groups of analyses separate. Whether the number of $\mathrm{Si}$ at॰ms and the total number of $\bullet$ tahedral positions filled are taken inte account (Figure 7) and the formulae are fitted as palygorskite, it is possible to find a continuous compositional variation which ranges between the richest in $\mathrm{Si}$ and lowest octahedral content, and the smallest Si contents and greater number of octahedral cations. Three groups of analyses can be separated. There are: (1) a cettain number of points with an excess - $\mathrm{Si}$ (>8 Si per half unit-cell); and (2) another group in which $>4.5 \bullet$ ctahedral positions are filled, that is t• say 'rioctahedral palygorskites'. They may correspond to sepielite when formulae are fitted to 32 exygens. (3) A third group (containing the most points) is characterized by a Si content between 7.5 and 8 , and between 4 and 4.5 -ctahedral positions filled. This group of points fits well with $\mathrm{M}$-rich palygorskite, as described by Chahi et al. (2002) and García-Romere et al. (2004). In Figure 7, if points that correspond to formulae fitted as sepiolite are analyzed, a continuous compositional variation can als•

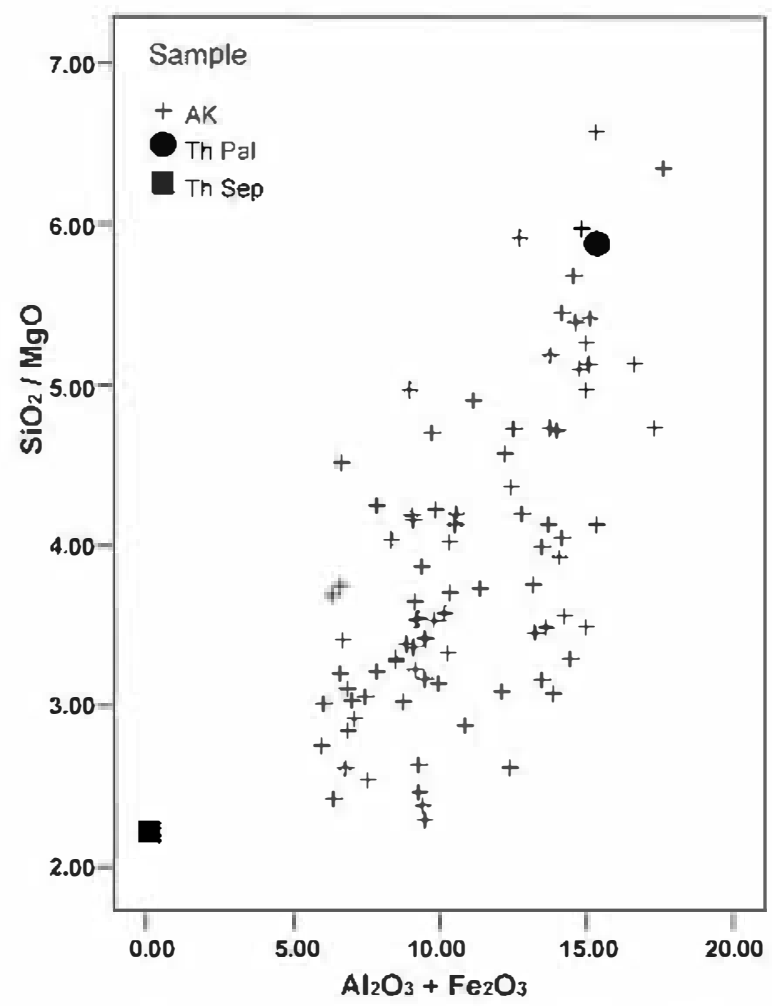

Figure 6. Chemical compesition (rati $\mathrm{SiO}_{2} / \mathrm{MgO} v \mathrm{~s} .\left(\mathrm{Al}_{2} \mathrm{O}_{3}+\right.$ $\left.\mathrm{Fe}_{2} \mathrm{O}_{3}\right)$ ) (\%) of the particles analyzed by AEM. Theoretical

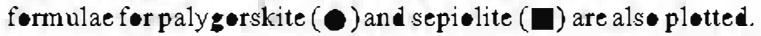

be found between two terms, these with excess Si $>12$ atoms per half unit-cell) and small octahedral content, and those with the smallest Si contents and the greatest number of octahedral cations. It is possible, nevertheless, to verify that there is no point that corresponds to sepiolite, and only a few of the points plotted are in the zone between 11.5 and $12 \mathrm{Si}$ atoms and 7-8 -ctahedral positions occupied.

$\mathrm{M}$ vs. $R 2 / R 3$ is plotted in Figure 8, fitted both as palygorskite and sepiolite formulae. In both cases, there is a continuous variation between the terms with greater $R 2 / R 3$ ratios and $\mathrm{M}$ content and those having lesser values of both variables. Most analyses fitted for 21 -xysens are projected in the field of palysorskite (taking inte account the bibliographic data mentioned above), that is to say, between 2 and 3.5 at $\bullet$ ranges between 1 and 3.5. However, as can be seen in the formulae fitted as palygorskite, most of the analyses correspond to Mg-rich palygorskite, because they are richer in $\mathrm{M}$ than ideal palygorskite. In contrast, none of analyses is plotted in the sepiolite field when the formulae are fitted to 32 oxygens. The field of sepiolite is plotted in Figure 7, in agreement with data published by Newman and Brown (1987) and Galán and Carreter (1999). Therefore, a field corresponding to sepiolite is plotted between 7 and $8 \mathrm{M}$ and $>7$ for $R 2 / R 3$. In fact, theoretical sepiolite could not be plotted on this graph because the rati $R 2 / R 3$ is equal to infinity in the ideal formula, in which all octahedral positions are occupied by $\mathrm{M} \mathbf{g}$, and $\mathrm{n} \bullet \mathrm{R}^{3+}$ cations $\left(\mathrm{Al} \bullet \mathrm{Fe}^{3+}\right.$ ) are present.

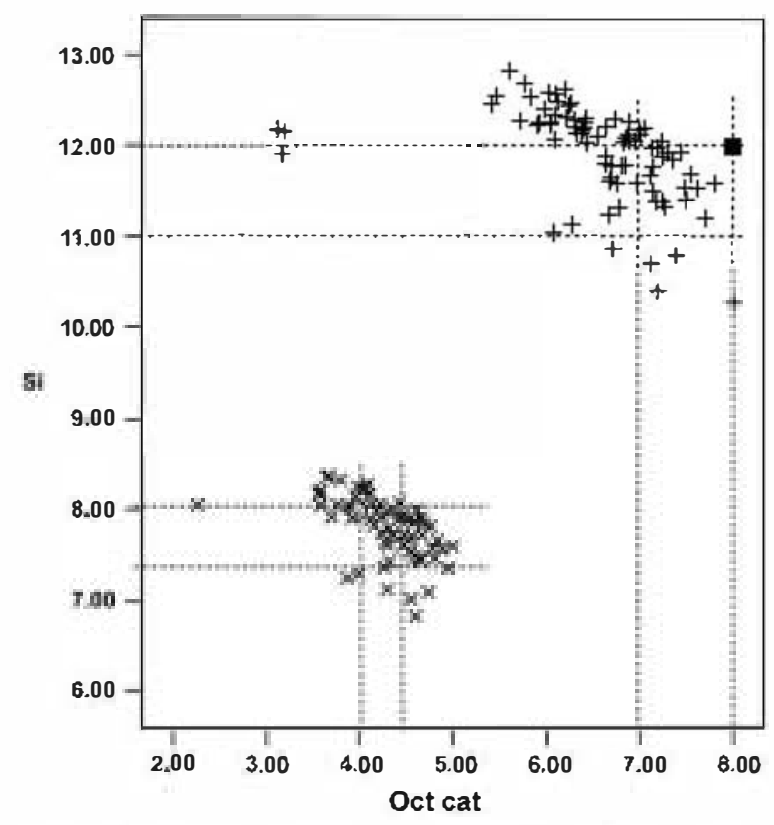

Figure 7. Number of Si at ms vs. number of ctahedral cations, per half unit-cell, calculated both as palyzorskite $(x)$ and as sepi lite $(+)$. Theoretical paly als• pletted. 


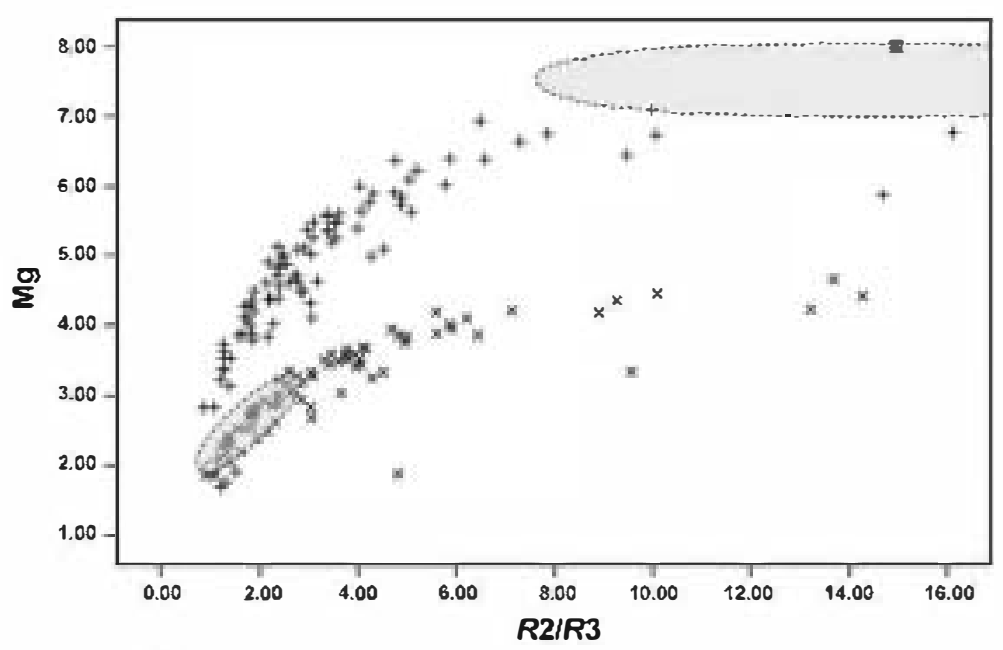

Figure 8. Mg vs $R 2 / R 3\left(\mathrm{Mg} /\left(\mathrm{A} 1+\mathrm{Fe}^{3+}\right)\right)$. All analyses are plotted, and fitted both as palygrskite $(\mathrm{x})$ and as sepi lite $(+)$. F $\bullet$ rmulae

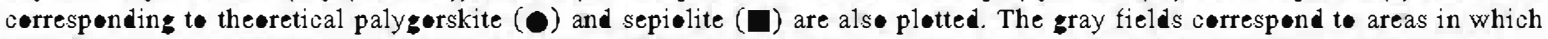
sepi lite could be prejected takin int accunt pessible is morphic substitution after lata reported in the literature (see text).

In Figure 8 none of the analyses is plotted in the sepiolite field but as sepiolite was determined by XRD in a propertion between $2-3 \%$, there should be a similar propertion in the analyzed fibers. All the AEM analyses have been carried out on is lated fibrous particles but, nevertheless, it is not possible to confirm that all of them correspend to pure palygorskite or pure sepiolite. Twe possibilities must be considered: (1) sepiolite from Allou Kagne depesit has an impertant number of isømerphic substitutions and is therefore an Al-rich sepiolite, or (2) analyses actually correspond to fibers of palygorskite and sepiolite together.

It is clear that there are fibers of pure palygorskite and pure sepilite, as can be seen by SAED (Figure 5a,b), and there are alsø particles formed by a mixture of sepiolite and palygorskite (Figure 5d). The is lated fibers that can be seen by TEM correspend to aggregates of several fibers of smaller size, and in this case they may be a mixture of crystals of sepiolite and palygorskite with different propertions of crystallites of both minerals. Although the possibility of the mixture of fibers of both minerals can be taken inte account, as mest of the fibers are monomineralic, then sepiolite must be very rich in $\mathrm{Al}$.

From the data set out above, some genetic considerations can be made. The Allou Kagne sepiolite-palygorskite was generated by sedimentation in an epicontinental marine environment. Sedimentary features of the levels studied have been seen in hand specimens and by SEM, and no evidence of diagenetic processes has been -bserved. Furthermore, there is evidence of authigenic formation of both sepiolite and palygorskite fibers. Therefore, palygorskite-sepiolite levels are the result of a chemical precipitation and the crystallochemical characteristics of the mineral particles should be a result - $f$ chemical composition of the solution. As the aggregates observed by SEM as small bundles are formed by very small numbers of fibers that can be either palygorskite, sepiolite, or both, this indicates the close genetic relationship between them, and epitactic growth may even be possible.

As has already been mentioned, it is not $\bullet$ ften that sepielite and palygorskite co-exist in nature. Experimental studies on the stability of fibrous clays show that sepiolite and/or palygorskite occurrences in sedimentary environments indicate saline conditions, with high activity of $\mathrm{Si}$ and $\mathrm{Mg}$ and high $\mathrm{pH}(8-10)$ (Siffert and Wey, 1962; Wøllast et al., 1968; La Iglesia, 1977). The formation of sepiolite or palygorskite depends on the availability of $\mathrm{Al}$ (Hay and Wiggins, 1980; Singer and Norrish, 1974). In the Allou Kagne deposit, palygorskite and sepiolite appear with X-ray amorphous silica. Direct precipitation of sepielite and palygorskite from solutions is more favered in the presence of X-ray amorphous silica than with quartz, and it is als favered by small values of $\mathrm{l} \bullet \mathrm{g}$ $\left.\left[\boldsymbol{a A l}{ }^{3+} /(\boldsymbol{a H})^{+}\right)^{3}\right]$ (Birsoy, 202).

Textural and microtextural features (Figure 4) allow us to propese that fibrous clay minerals of the Alløu Kagne depesit were formed by direct precipitation from solution. If both minerals precipitate together from the same solution, so close together that sometimes they comprise a single small bundle, this suggests that they grow at the same time and therefore formation conditions were very reswicted, close to the limit of their stability fields. Lower aqueous Al activities favor the non-Al phases (sepiolite) with respect to the Al-containing phases (palygorskite). At lower $\mathrm{pH}$ values, palygorskite can be formed by the transformation of the amorpheus silica and diectahedral smectites. At slightly higher $\mathrm{pH}$ values, sepiolite, amorphous silica and palygorskite can form from the solution. In silica-poor solutions the formation of sepiolite requires a higher $\mathrm{pH}$ than that of palygorskite. Concentrated silica solutions 
$\left(\log \left[a \mathrm{H}_{4} \mathrm{SiO}_{4}\right]>-4.75\right)$ but lower Al activities are the most favorable conditions for the direct precipitation $\bullet$ sepiølite frøm sølution (Birsøy, 2002).

The chemical compesition of the selution favered the formation of palygerskite rather than sepiølite, due to the presence of reactive $\mathrm{Al}$ in the solution with significant values of $\mathrm{Mg}$ and $\mathrm{Si}$ activity. A wide compesitional variation of palygorskites and sepielites (from palygerskites close to ideal formula to $\bullet$ thers very rich in $\mathrm{Mg} \bullet \mathrm{Al}$ ) appear as a consequence •f temp•rary variations of the chemical compesition of the sølution. These compesitional variations could be a consequence - cyclical variations in the formation of palygerskite which consumes Al. The variations in the compesition of the sølutions could alsø be influenced by the formation - intermediate celleidal phases as ultrafine aluminøus cølløids. When palygerskite forms, Al activity is reduced, and the new conditions faver the precipitation

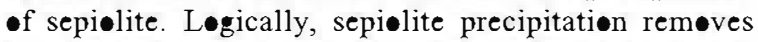
$\mathrm{Mg}$ from the sølution and increases Al activity, and a new cycle begins with new precipitation of palygerskite. Both phases can even form in the same cycle, due to the proximity of the tw phases in the stability diagrams,

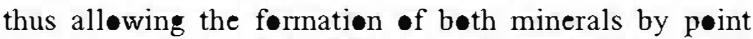
changes in the micrechemical conditions.

Palygerskites in calcareøs formations are eccasionally mixed with smectites. The ectahedral compesition -f smectites and fibrous clays partly $\bullet$ verlap. Sepi lite is clearly in the triectahedral demain but the palygerskite field is both in the diectahedral domain as well as between the diectahedral and triectahedral domains of smectites (Paquet et al., 1987). However, it is clear that smectites mixed with the fibrous clays do not appear in the Alleu Kagne depesits, suggesting a relatively mere alkaline, siliceøus and magnesic envirønment compared to that which is necessary for the formation of smectite, but nøt necessarily møre saline (Jønes and Galán, 1988). The increased evaporation of water, together with an increase in alkali activity and in $\mathrm{pH}$, would faver the formation of mixed-layer kerelite-stevensite. The tri-ctahedral smectite forms in the same system at higher $\mathrm{pH}$ (Khøury et al., 1982). Precipitation of sepiølite and/ -r palygerskite depended en evaperation, and on rain and fresh-water flows that temperarily changed the $\mathrm{pH}$ in søme parts of the closed basin. Semi-arid climatic conditions interrupted by humid intervals prevailed, and these did not allow the develøpment of evaporitic facies

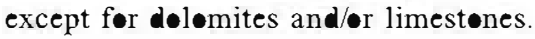

\section{ACKN WLEGMENTS}

The authors are grateful to Adrian Gómez Herrero of the Centro de Microscopía Electrónica 'Luis Bru (U.C.M.), and to Catherine Doyle for checking and improving the English. The work benefited from helpful comments by $\mathbf{D r}$ Blair Jones and Dr Crawford Elliott. Financial support by CICYT (project CGL2006-09843/BTE) is acknowledged. Additional funding was obtained from Grant 910386 (Universidad Complutense, Grupos de Investigación)

\section{REFERENCES}

Akbulut, A. and Kadir, S. (2003) The geology and origin of sepiolite, palygorskite and saponite in Neogene lacustrine sediments of the Serinhisar-Acipayam Basin, Denizly, SW Turkey. Clays and Clay Minerals, 51, 279-292.

Alvarez, A. (1984) Sepiolite properties and uses. Pp. 253-287 in: Palygørskite-Sepiølite. Occurrences, Genesis and Uses (A. Singer and E. Galán, editors). Developments in Sedimentology, 37. Elsevier, Amsterdam.

Bailey, S.W. (1980) Structure of layer silicates. Pp. $1-123$ in: Crystal Structures of Clay Minerals and their X-ray Identification (G.W. Brindley and G. Brown, editors). Monograph 5, Mineralogical Society, London.

Birsoy, R. (2002) Formation of sepiolite-palygorskite and related minerals from solution. Clays and Clay Minerals, 50, $736-745$.

Bowles, A., Angino, E.A., Hosterman, J.W. and Galle, O.K. (1971) Precipitation of deep-sea palygorskite and sepiolite Earth and Planetary Science Letters, 11, 324-332.

Bradley, W.F. (1940) The structural scheme of attapulgite American Mineraløgist, 25, 405-411.

Brauner, K. and Preisinger A. (1956) Estruktur und Enstehung des sepiolits. Tschermaks Mineraløgische und Petr@graphisches Mitteilungen, 6, 120-140.

Castillo, A. (1991) Geología de los yacimientos de minerales del grupo paligorskita-sepiolita. Pp. 609-636 in:

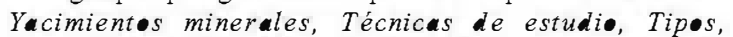
Evoloción metaløgnética, Expløación (R. Lunar and R. Oyarzun, editors). Editorial Centro de Estudios ramón Areces, S.A., Spain.

Chahi, A., Fritz, B., Duplay, J., Weber, F. and Lucas, J. (1997) Textural transition and genetic relationship between precursor stevensite and sepiolite in lacustrine sediments (Jbel Rhassoul, Morocco). Clays and Clay Minerals, 45, 378-389.

Chahi, A., Petit, S. and Decarreau, A. (2002) Infrared evidence of dioctahedral-trioctahedral site occupancy in palygorskite. Clays and Clay Minerals, 50, 306-313.

Estéoule-Choux, J. (1984) Palygorskite in the Tertiary deposits of the Armorican Massif. Pp. 75-85 in: PalygrorskiteSepiolite. Occurrences, Genesis and Uses (A. Singer and E. Galán, editors) Developments in Sedimentology, 37. Elsevier, Amsterdam.

Galán, E. and Carretero, I. (1999) A new approach to compositional limits for sepiolite and palygorskite. Clays and Clay Minerals, 47, 399-409.

Galán, E. and Castillo, A. (1984) Sepiolite-palygorskite in Spanish Tertiary basins: Genetical patterns in continental environments. Pp. 87-124 in: Palygørkite-Sepiølite. - ccurrences, Genesis and Uses (A. Singer and E. Galán, editors). Developments in Sedimentology, 37. Elsevier, Amsterdam.

Galán, E. and Ferrero, A. (1982) Palygorskite-sepiolite clays of Lebrija, Southern Spain. Clays and Clay Minerals, 30, 191-199.

Garcia-Romero, E., Suárez Barrios, M. and Bustillo Revuelta, M.A. (2004) Characteristics of a Mg-palygorskite in Miocene rocks (Madrid Basin, Spain). Clays and Clay Minerals, 52, 486-494.

Garcia-Romero, E., Suárez, M., Oyarzum, R., López-García, J.A. and Regueiro, M. (2006) Fault-hosted palygorskite from the Serrata de Nijar deformation zone (SE Spain). Clays and Clay Minerals, 54, 324-332.

Haji-Vassilou, A. and Puffer, J.H. (1975) A macrocrystalline attapulgite-palygorskite ocurrence in calcite veins. American Mineralogist, 60, 328-330.

Hay, R.L. and Wiggins, B. (1980) Pellets, ooids, sepiolite and silica in three calcretes of the southwestern United States. 
Sediment•løgy, 27, 559-576.

Jones, B.F. and Galán, E. (1988) Sepiolite and palygorskite. Pp. 631-674 in: Hydrous Phyllosilicates (exclusive of micas) (S.W. Bailey, editor). Reviews in Mineralogy, 19. Mineralogical Society of America, Washington, D.C.

Kamineni, D.C., Griffault, L.Y. and Kerrich, R. (1993) Palygorskite from fracture zones in the Eye-Dashwa Lakes granitic pluton, Atikokan, Ontario. The Canadian Mineraløgist, 31, 173-183.

Khoury, J.N., Eberl, D.D. and Jones, B.F. (1982) Origin of magnesium clays from the Amargosa Desert, Nevada. Clays and Clay Minerals, 30, 327-336.

La Iglesia, A. (1977) Precipitación por disolución homogénea de silicatos de aluminio y magnesio a temperatura ambiente.

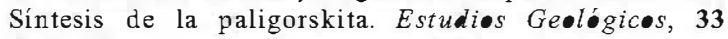
535-544.

Leguey, S., Martín Rubí, J.A., Casas, J., Marta, J., Cuevas, J., Álvarez, J. and Medina, J.A. (1995) Diagenetic evolution and mineral fabric in sepiolitic materials from the Vicalvaro Deposit (Madrid Basin). Pp. 383-392 in: Proceedings of the 10th International Clay Conference, Adelaide.

Lopez-Galindo, A., Ben Aboud, A., Fenoll Hach-Ali, P. and Casas Ruiz, J. (1996) Mineralogical and geochemical characterization of palygorskite from Gabasa (NE Spain). Evidence of a detrital precursor. Clay Minerals, 31, 33-44.

Martín Pozas, J.M., Sánchez Camazano, M. and Martín Vivaldi Martínez, J.L. (1981) La Paligorskita de Tabladillo

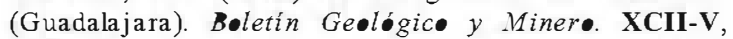
$395-402$.

Martín Vivaldi, J.L. and Cano, J. (1956) Contribution to the study of sepiolite: II Some considerations regarding the mineralogical formula. Pp: 173-176 in: Proceedings of the furth National Conference on Clays and Clay Minerals. National Academy of Sciences - National Research Council Publication 456, 1956, USA.

Millot, G. (1970) Ge•løgy of Clays: Weathering, Sediment•løgy, Geøchemisty. Springer Verlag, New York, $430 \mathrm{pp}$.

Newman, A.C.D. and Brown, G. (1987) The chemical constitution of clays. Pp. 1-128 in: Chemistry of Clays and Clay Minerals (A.C.D. Newman, editor). Monograph 6, The Mineralogical Society, Longman Scientific and Teclinical, Harlow, Essex, UK.

Paquet, H., Duplay, J., Vallerçon-Blanc, M.M. and Millot, G. (1987) Octahedral compositions of individual particles in smectite-palygorskite and smectite-sepiolite assemblages. Pp. 73-77 in: Proceedings of the International Clay Conference, Denver, 1985 (L.G. Schultz, H. Van Olphen and F.A. Mumpton, editors), The Clay Minerals Society, Bloomington, Indiana.

Serna, C., Van Scoyoc, G.E. and Ahlrichs, J.L. (1977)
Hydroxyl groups and water in palygorskite. American Mineral•gist, 62, 784-792.

Siffert, B. and Wey, R. (1962) Synthèse d'une sépiolite à température ordinaire. Comptes rendus de l'Academie des sciences, Paris, 245, 1460-1463.

Singer, A. (1984) Pedogenic palygorskite in the arid environment. Pp. 169-175 in: Palygerskite-Sepi-lite. Occurrences, Genesis and Uses (A. Singer and E. Galán, editors). Developments in Sedimentology, 37. Elsevier, Amsterdam.

Singer, A. and Norrish, K. (1974) Pedogenetic palygorskite. Occurrences in Australia. American Mineralegist, 59, $508-517$.

Suárez, M., Robert, M., Elsass, F. and Martín Pozas, J.M. (1994) Evidence of a precursor in the neoformation of palygorskite - New data by analytical electron microscopy. Clay Minerals, 29, 255-264.

Tien, P.L. (1973) Palygorskite from Warren Quarry, Enderby, Leicestershire, England. Clay Minerals, 10, 27-34

Torres-Ruiz, J., López-Galindo, A., González-López, J.M. and Delgado, A. (1994) Geochemistry of Spanish sepiolitepalygorskite deposits: Genetic considerations based on trace

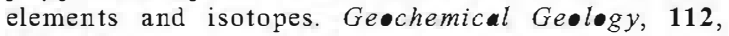
$221-245$.

Verrecchia, E.P. and Le Coustumer, M.N. (1996) Occurrence and genesis of palygorskite and associated clay minerals in Pleistocene calcrete complex, Sde Boqer, Negev desert, Israel. Clay Minerals, 31, 183-202.

Watts, N.L. (1976) Paleopedogenic palygorskite from the basal Permo-Triassic of northwest Scotland. American Mineraløgist, 61, 299-302.

Watts, N.L. (1980) Quaternary pedogenic calcretes from the Kalahari (southern Africa): mineralogy, genesis and diagenesis. Sediment•løgy, 27, 661-686.

Weaver, C.E. (1984) Origin and geologic implications of the palygorkite of S.E. United States. Pp. 39-58 in: Palygerskite-Sepiolite. occurrences, Genesis and Uses (A. Singer and E. Galán, editors). Developments in Sedimentology, 37. Elsevier, Amsterdam.

Wollast, R., Mackenzie, F.T. and Bricker, O. (1968) Experimental precipitation and genesis of sepiolite at earth-surface conditions. American Mineral•gist, 53, $1645-1662$.

Yaalon, D.M and Wieder, M. (1976) Pedogenetic palygorskite in some arid brown (calciothid) soils of Israel. Clay Minerals, 11, 73-79.

Zaaboub, N., Abdel japouad, S. and López Galindo, A. (2005) Origin of fibrous clays in Tunisian Paleogene continental deposits. Journal of African Earth Sciences, 43, 491-504. 